\title{
A case report of tinea capitis in infant in first year of life
}

Narcisa Mandras ${ }^{1 \dagger}$, Janira Roana ${ }^{1 \dagger}$, Ornella Cervetti ${ }^{2}$, Michele Panzone ${ }^{2}$ and Vivian Tullio ${ }^{1 *}$ (D)

\begin{abstract}
Background: Tinea capitis is a cutaneous fungal infection common among 3 to 7 year old children but it is rare in the first year of life.

Case presentation: We present a case of a 12-month-old infant with erythematous scalp lesions combined with hair loss. He was suspected of dermatophytosis and mycological analysis of all suspected lesions was performed. Clinical features and culture results confirmed tinea capitis caused by Microsporum canis. The infant patient was treated with griseofulvin for 2 months. However, 15 days later at the end of treatment he presented with a single vesicle positive for M. canis. Griseofulvin therapy continued for another month. After 3 months of follow-up, no recurrence was observed.

Conclusions: In infant, sometimes tinea capitis is misdiagnosed and underreported because it is similar to other scalp pathologies. Therefore, if erythematous scalp lesions are present, they must be examined from a mycological point of view to inform the differential diagnosis. Once diagnosed, treatment of tinea capitis can pose a dilemma because different factors may influence the choice between equally effective therapies (i.e. safety, age, formulation, cost). This case report suggests that it is important to establish an accurate diagnosis and treatment for this dermatophytosis to avoid recurrences or therapeutic failures, especially in infants.
\end{abstract}

Keywords: Pediatric infections, Case report, Tinea capitis, Dermatophytes, Microsporum canis, Correct treatment

\section{Background}

Tinea capitis (TC) is a fungal infection of the scalp and the surrounding skin due to dermatophytes such as Microsporum spp. and Trichophyton spp. [1, 2]. It is a predominantly dermatophyte infection in children 3 to 7 years old and it is rare in infants in the first year of life [3, 4]. We report a case of an infant with TC admitted to the Medical Science Department, University of Turin (Italy).

\section{Case presentation}

A.M., a 12-month-old male infant, Caucasian, Italian, in good general health, with no history of recent fever or any other symptoms. The patient was evaluated for erythematous scalp lesions and annular patches combined with hair loss (Fig. 1). The infant had not been in contact

\footnotetext{
* Correspondence: vivian.tullio@unito.it

†Narcisa Mandras and Janira Roana contributed equally to this work.

${ }^{1}$ Department of Public Health and Pediatrics, University of Turin, Torino

10126, Italy

Full list of author information is available at the end of the article
}

with animals; her mother and other family members were asymptomatic. No other systemic symptoms were elicited. He was suspected to have a dermatophytosis.

The Wood's light examination revealed a brilliant green fluorescence on the scalp lesions. Mycological analysis of all suspected dermatophyte lesions was performed. Hair and scale samples were collected and examined under a light microscope with $20 \% \mathrm{v} / \mathrm{v}$ $\mathrm{KOH}+40 \% \mathrm{v} / \mathrm{v}$ DMSO solution in distilled water. A fungal culture was performed into Mycobiotic agar (Merck, KGAA, Germany) to identify dermatophytes. Plates were incubated at $25^{\circ} \mathrm{C}$ and examined every $2-3$ days for at last 15 days. Mold identification was based on macroscopic and microscopic assessment of colonies [5]. Macroscopic examination revealed some white fluffy spreading colonies (Fig. 2), and a characteristic deep yellow-orange pigment on the reverse. Spindle shaped multicellular macroconidia with thick cell walls were detected on microscopic examination (Fig. 3). Clinical features and culture results reveled TC caused by Microsporum canis. 


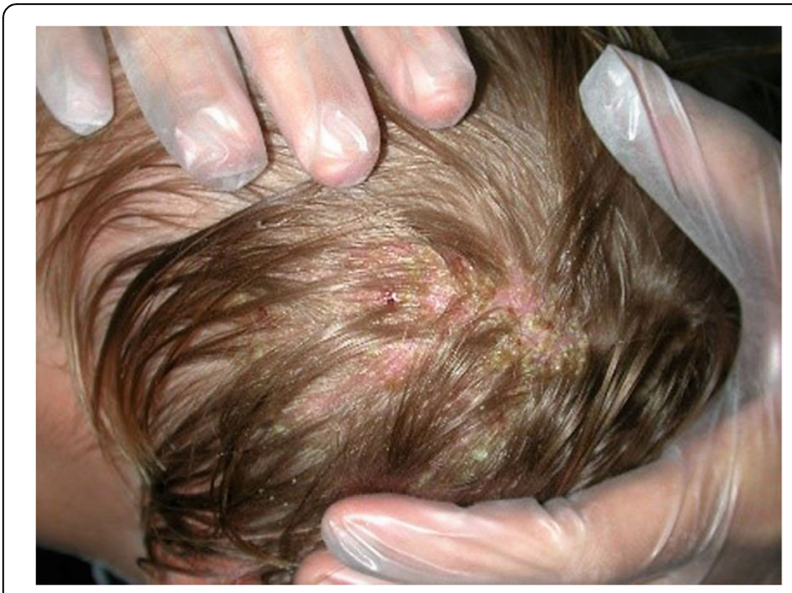

Fig. 1 A 12 month-old male infant presented a history of erythematous scalp lesions combined with hair loss

Therapy was started with oral griseofulvin $(20 \mathrm{mg} / \mathrm{kg} /$ day $)$ with a 2 daily tioconazole cream application. The lesions were also treated with iodized alcohol. At 15-day intervals, the child was subjected to objective examination, including culture tests. The patient was treated for two months in total with both medications and iodized alcohol. After this period of treatment, the first negativization of the culture for $M$. canis was observed. However, 15 days later at the end of treatment, in the area of the lesions, where hair regrowth was observed, the infant patient presented with a single vesicle with growth of M.canis on culture. Oral treatment with griseofulvin $(20 \mathrm{mg} / \mathrm{kg} /$ day $)$ was administered for one month. After this period, all scalp lesions were completely healed and cultures resulted negative for dermatophytes. After 3 months of follow-up, no recurrence was observed (Additional file 1).

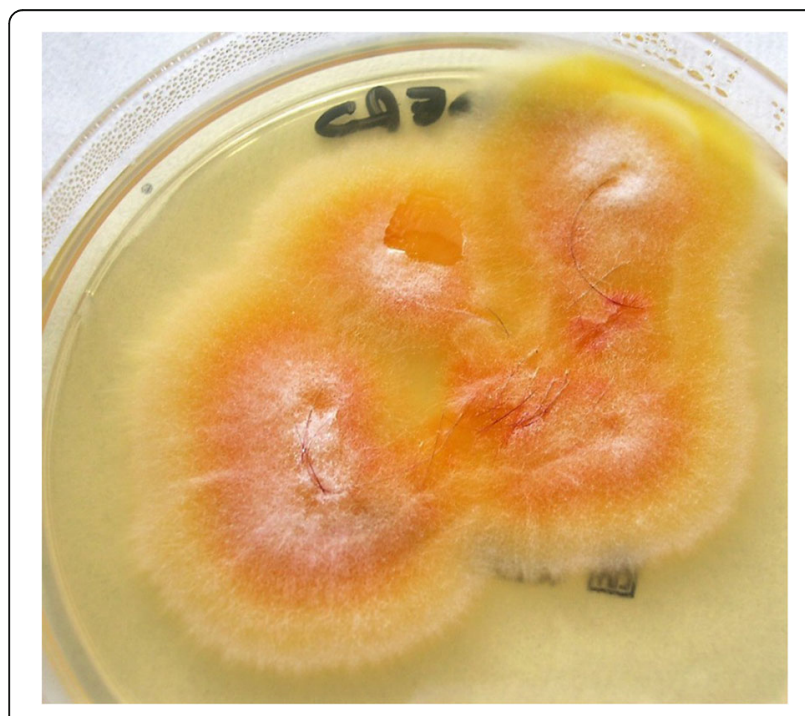

Fig. 2 Culture of the specimen revealed the presence of $M$. canis colonies

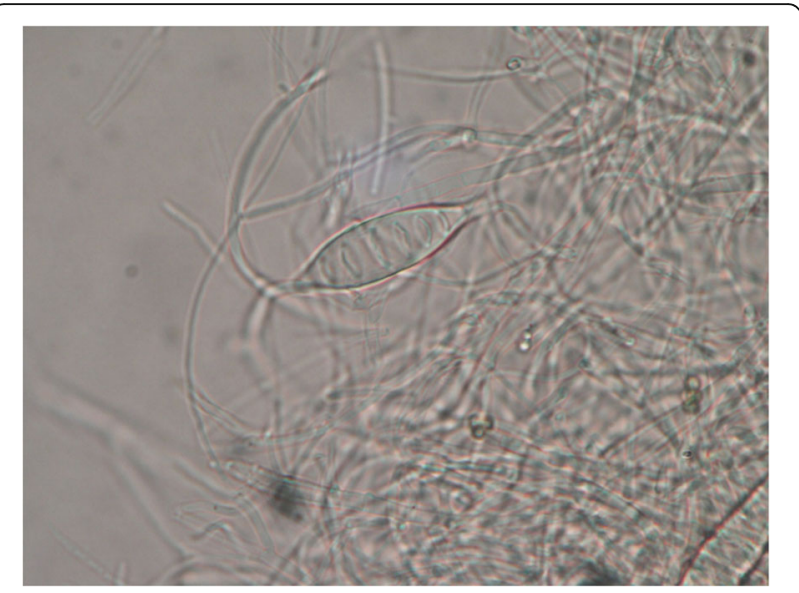

Fig. 3 "Spindle" shaped multicellular macroconidia (400x)

\section{Discussion and conclusions}

Epidemiology of TC can be related to geographical location and social, cultural and nutritional factors: in infants, across Europe and the Mediterranean basin, $M$. canis remains the most common organism responsible for $\mathrm{TC}$, with prevalence ranges between 0.23 and 2.6\%. Trichophyton tonsurans is reported as accounting for $50-90 \%$ of dermatophyte scalp isolates in the UK and the USA. In addition, $T$. tonsurans has spread to both South America and West Africa. T.violaceum is the most common in Greece and Belgium [6-8].

Tinea capitis in infants likely is more common than is reported and recent literature demonstrates a significant increase. Although the incidence is low, sometimes TC is misdiagnosed and underreported, and differential diagnosis may include seborrheic dermatitis, atopic dermatitis, neonatal lupus, Langerhans cell histiocytosis and syphilis. TC should be suspected in a child with alopecia, pruritus and/or persistent desquamation and thinning hair, and the scalp lesion should be investigated from a mycological point of view $[6,9,10]$.

The drug of choice for the treatment of TC is griseofulvin in children. Six to twelve weeks therapy is recommended or until the patient tests negative for fungi (light microscopy and culture). However, the long period of treatment required with this antifungal drug is a significant disadvantage and leads to reduced compliance [8]. Other oral antifungals, specifically fluconazole, itraconazole, ketoconazole, and terbinafine are available and give the advantage of good safety and efficacy profiles, and shorter required duration of treatment of TC caused by Tricophyton and Microsporum. [8]. Fremerey and Nenoff reported a case of TC in a newborn caused by T. soudanense [11]. The patient was initially treated with intravenous fluconazole and topical clotrimazole and octenidine therapy; after one week of treatment, the lesions were resolving [11]. Oral terbinafine, which is often 
administered in dermatophytosis, is FDA approved only for treatment of TC in children four years of age or older. Oral ketoconazole has been suggested for treatment of dermatophyte infections where griseofulvin is not tolerated. However, Michaels et al. do not consider oral ketoconazole to be as prudent an alternative due to the higher risk of hepatotoxicity [3].

Nevertheless griseofulvin is the cheapest agent in tablet form, has the last potential drug interactions, and is available as a suspension. Among all the alternative drugs to griseofulvin for the treatment of TC, the most promising, as reported by Shemer et al. [12], is fluconazole; in fact, the azole was found in children to have efficacy similar to that griseofulvin in infection due to $M$. canis, $T$. verrucosum and $T$. violaceum. This drug also reaches high concentrations in the epidermis and persists for several weeks, and is optimal for children because it is supplied as a rectal preparation or syrup and can be used only once weekly [12].

Binder et al. reported that TC caused by Microsporum species may be treated with a longer duration of itraconazole and $5 \mathrm{mg} / \mathrm{kg} /$ day is used for infantile superficial fungal infections within 3 to 6 weeks [13]. However, griseofulvin remains the drug of choice in infants, because it is efficacious (it can penetrate the hair sheath well), it has lower cost and excellent safety profile and continues to be the better treatment option for TC due to $M$. canis $[3,4,6,12]$.

In the present case report, the patient received oral griseofulvin with a daily wash using tioconazole cream application. The therapy lasted for 3 months with a recurrence, probably indicating a low efficacy of griseofulvin at the doses administered. However, it would be important to understand that there are benefits to the use of other antifungal drugs such as fluconazole and itraconazole in comparison with griseofulvin.

In conclusion, although we have presented a single case of TC by $M$. canis in a 12-month-old infant, this may suggest that it is important to establish an accurate diagnosis and treatment for this dermatophytosis to avoid recurrences or therapeutic failures, especially in infants.

\section{Additional file}

Additional file 1: Timeline. Relevant medical history and interventions, in a 12-month-old male infant, are organized as a timeline. The patient was admitted to the Medical Science Department, University of Turin (Italy) and he was evaluated for erythematous scalp lesions and annular patches combined with hair loss. (DOCX $33 \mathrm{~kb}$ )

\section{Abbreviations}

DMSO: Dimethyl sulfoxide; KOH: Potassium hydroxide; TC: Tinea capitis

Acknowledgements

Not applicable.
Funding

None.

\section{Availability of data and materials}

The data that support the findings of this study are available from the corresponding author upon reasonable request.

\section{Authors' contributions}

MN, PM, CO, RJ and TV made substantial contributions to conception and design, analysis and interpretation of data. MN, PM and RJ have been involved in acquisition of data: laboratory and clinical. MN and TV reviewed the literature, drafted the manuscript. PM been involved in revising it critically for important intellectual content. TV took the pictures of M.canis. TV is the corresponding author. All authors read and approved the final manuscript. They given final approval of the version to be published

Ethics approval and consent to participate

Not applicable.

\section{Consent for publication}

Written informed consent from the parents of the patient was obtained for the publication of this Case Report and accompanying images.

\section{Competing interests}

The authors declare that they have no competing interests.

\section{Publisher's Note}

Springer Nature remains neutral with regard to jurisdictional claims in published maps and institutional affiliations.

\section{Author details}

${ }^{1}$ Department of Public Health and Pediatrics, University of Turin, Torino

10126, Italy. ${ }^{2}$ Department of Medical Sciences, University Clinic of

Dermatology, University of Turin, Torino, Italy.

Received: 16 November 2018 Accepted: 12 February 2019

Published online: 22 February 2019

\section{References}

1. Kundu D, Mandal L, Sen G. Prevalence of tinea capitis in school going children in Kolkata, West Bengal. J Nat Sci Biol Med. 2012;3:152-5.

2. Farooqi M, Tabassum S, Rizvi DA, Rahman A, Rehanuddin AS, Mahar SA Clinical types of tinea capitis and species identification in children: an experience from tertiary care centres of Karachi, Pakistan. J Pak Med Assoc 2014;64:304-8.

3. Michaels BD, Del Rosso JQ. Tinea capitis in infants: recognition, evaluation, and management suggestions. J Clin Aesthet Dermatol. 2012;5:49-59.

4. Adefemi SA, Odeigah LO, Alabi KM. Prevalence of dermatophytosis among primary school children in Oke-Oyi community of Kwara state. Niger J Clin Pract. 2011;14:23-8.

5. Tullio V, Cervetti O, Roana J, Panzone M, Scalas D, Merlino C, Allizond V, Banche G, Mandras N, Cuffini AM. Advances in Microbiology, Infectious diseases and public health: refractory Trichophyton rubrum infections in Turin, Italy: a problem still present. Adv Exp Med Biol. 2016;901:17-23.

6. Atanasovski M, El Tal AK, Hamzavi F, Mehregan DA. Neonatal dermatophytosis: report of a case and review of the literature. Pediatr Dermatol. 2011:28:185-8.

7. Mashiah J, Kutz A, Ben Ami R, Savion M, Goldberg I, Gan Or T, Zidan O, Sprecher E, Harel A. Tinea capitis outbreak among paediatric refugee population, an evolving healthcare challenge. Mycoses. 2016;59:553-7.

8. Bennassar A, Grimalt R. Management of tinea capitis in childhood. Clin Cosmet Investig Dermatol. 2010:3:89-98.

9. Gilaberte Y, Rezusta A, Gil J, Sáenz-Santamaría MC, Coscojuela C, Navarro M, Zubiri ML, Moles B, Rubio MC. Tinea capitis in infants in their first year of life. Br J Dermatol. 2004;151:886-90.

10. Zampella JG, Kwatra SG, Blanck J, Cohen B. Tinea in tots: cases and literature review of oral antifungal treatment of tinea capitis in children under 2 years of age. J Pediatr. 2017;183:12-8.

11. Fremerey C, Nenoff P. Tinea capitis in a newborn. N Engl J Med. 2018;378:2022. 
12. Shemer A, Grunwald MH, Gupta AK, Lyakhovitsky A, Daniel CR 3rd, Amichai B. Griseofulvin and fluconazole reduce transmission of tinea capitis in schoolchildren. Pediatr Dermatol. 2015;32:696-700.

13. Binder B, Richtig E, Weger W, Ginter-Hanselmayer G. Tinea capitis in early infancy treated with itraconazole: a pilot study. J Eur Acad Dermatol Venereol. 2009;23:1161-3.

Ready to submit your research? Choose BMC and benefit from:

- fast, convenient online submission

- thorough peer review by experienced researchers in your field

- rapid publication on acceptance

- support for research data, including large and complex data types

- gold Open Access which fosters wider collaboration and increased citations

- maximum visibility for your research: over $100 \mathrm{M}$ website views per year

At BMC, research is always in progress.

Learn more biomedcentral.com/submissions 